\title{
(1) \\ ADVANCING THE SCIENCE OF SELF-MANAGEMENT IN ADULTS WITH LONG-TERM \\ LEFT VENTRICULAR ASSIST DEVICES
}

Casida, Jesus (proxy) (contact); Aikens, James; Pagani, Francis; Ewald, Gregory;

Craddock, Heidi; Pavol, Marykay; Schroeder, Sarah; Yang, James

Corresponding Author

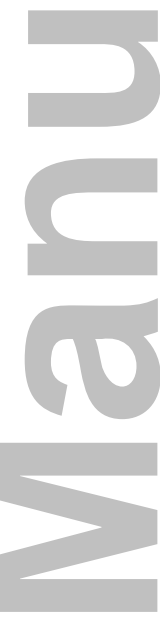

Jesus Casida

University of Michigan - School of Nursing

426 North Ingalls Room 4138 Ann Arbor Michigan 48109

United States

T: 313-204-7665 F: (734) 936-5525

Thoughts \& Progress

Received: September 23, 2017

Revised: November 14, 2017

This is the author manuscript accepted for publication and has undergone full peer review but has not been through the copyediting, typesetting, pagination and proofreading process, which may lead to differences between this version and the Version of record. Please cite this article as doi:10.1111/ aor.13113. 


\section{Abstract}

This study tested the applicability of the Individual and Family Self-Management Theory (IFSMT) to self-management (SM) in patients with left ventricular assist devices (LVADs).

From an existing data set, we extracted the following variables that correspond to IFSMT's conceptual dimensions: anxiety, depression, and cognition [context dimension]; self-efficacy [SM process dimension]; adherence and quality of life (QOL) [outcome dimensions].

Descriptive statistics and partial least squares path modeling procedures were used for data analyses. A total of 100 patients (mean age $52 \pm 13.4$ years) with continuous flow LVAD designs comprised the present study. Most patients were White (78\%), married (69\%), college-educated $(72 \%)$, and on disability (53\%). Their mean anxiety and depression scores were slightly above normal, while their cognitive function scores were slightly lower than normal. LVAD care self-efficacy, adherence, and QOL were within normal ranges. Factor loadings ranged from .50 to 1.0 , and there were significant forward path relationships among the context, process, and outcome dimensions ( $\beta$ ranges from .02 to .60, all $p$ values < .05). In conclusion, the IFSMT provides a good fit for SM in LVAD. Further research is needed to clarify how best to improve LVAD SM practice and treatment outcomes.

Key words: left-ventricular assist devices, circulatory support, self-management, self-management theory, self-management of implantable artificial organs

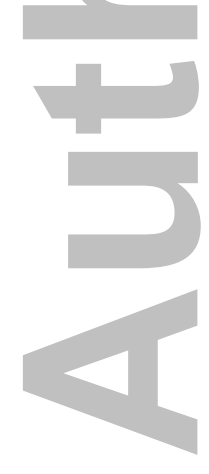




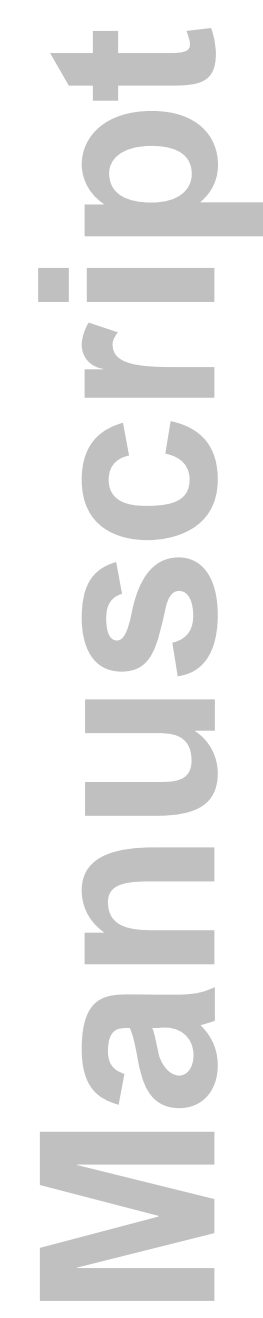

ADVANCING THE SCIENCE OF SELF-MANAGEMENT IN ADULTS WITH LONG-TERM

\section{LEFT VENTRICULAR ASSIST DEVICES}

\section{Background}

Over the past two decades, the survival and quality of life (QOL) of patients suffering from advanced heart failure have been remarkably improved by implantable left ventricular assist devices (LVADs). This improvement in outcomes was attributed in part to the advancements in technology, surgical techniques, and post-operative management. ${ }^{1,2}$ However, despite the miniaturization and simplification of LVAD designs, the selfmanagement (SM) of the LVAD care regimen post hospital discharge is still excessively 
complex for many patients. ${ }^{3}$ The LVAD care regimen consists of several tasks and procedures performed by patients on a daily basis to maintain the normal workings of the LVAD system, prevent complications, reduce heart failure symptom burden and rehospitalization, and to ultimately optimize health and QOL. ${ }^{3-5}$

During the first 6 months post discharge, most patients require assistance from family caregivers to manage the LVAD care regimen, as a result of their functional restriction, cognitive difficulty, and/or frailty. ${ }^{3,5}$ Enlistment of family caregivers in SM and available support from VAD nurses/coordinators ${ }^{6}$ is a healthcare delivery model aligned with the contemporary conceptualization of SM in high acuity and chronically ill patients. ${ }^{7,8}$ Studies have shown that nurse-supported SM interventions, along with healthcare providersupported SM programs in adults with multiple comorbidities, are associated with improved health and QOL, as well as reduced hospital readmission and mortality rates. ${ }^{8-10}$

$\mathrm{SM}$ is a multidimensional construct, interchangeably referred to as "self-care" in the mechanical circulatory support literature. ${ }^{3}$ Self-care is conceptually related to SM, but there is a growing consensus in the health sciences literature clarifying the distinction between the two. Simply defined, self-care is used to describe health promotion and risk reduction behaviors (eg, proper nutrition, exercise, and sleep) performed by individuals without assistance from family caregivers and/or guidance from licensed healthcare professionals, whereas SM is performed by individuals with assistance.${ }^{11-13}$ Unfortunately, both SM and self-care remain understudied in LVAD, despite the customary practice of patients and/or family caregivers being held responsible for LVAD care management post hospital discharge. ${ }^{5,14}$

To address the dearth of scientific knowledge of LVAD SM, we identified a theory that can be used by scholars and clinicians alike as a guiding framework for LVAD SM research and practice. This paper describes both a theory and a study aimed at explicating 
the conceptual elements or dimensions of the theory in adults with long-term LVADs. Future directions and applications of the theory in LVAD SM research and practice are discussed. LVAD SM Theoretical Framework

The Individual and Family Self-Management Theory (IFSMT) ${ }^{8,15}$ is a middle range descriptive theory that has been widely used in research involving adults with chronic and multiple conditions. The IFSMT describes SM as a complex phenomenon with three distinct but interconnected dimensions: context, process, and outcomes (Figure 1). ${ }^{15}$ The context dimension describes SM risks and protective factors at the individual or family level. For example, cognitive dysfunction, an absence of family caregivers, or lack of social support are all considered risk factors of an effective LVAD SM. On the contrary, sufficient LVAD care education and competent caregivers are protective factors of LVAD SM. VAD nurses/coordinators ${ }^{6}$ can be added as SM protective factors, as they provide continual SM support for LVAD care in the out-patient setting. ${ }^{6}$ The process dimension refers to elements of SM including self-regulation (eg, health behavior change), self-efficacy, goal-setting, and others. Finally, the outcome dimension consists of proximal and distal outcomes. The proximal outcome is the actual engagement (eg, treatment adherence) or direct result of the SM process, which in turn determines the distal outcomes (eg, QOL). ${ }^{8,15}$

The multidimensional constructs of the IFSMT are organized as a list of concepts clustered in each theoretical dimension, shown in Figure $1 .{ }^{15}$ The lines and arrows in this figure illustrate the underlying theoretical assumption that if context and/or SM process variable(s) is/are changed (eg, interventions at individual and/or family level), then proximal and/or distal outcome dimension(s) is/are also expected to change. This assumption is supported by causal inference and association studies in adults with complex chronic diseases, $8,10,15$ but has yet to be tested in the LVAD population. Thus, we examined the 
relationships of variables operationalizing theoretical dimensions (context, process, and outcome) of the IFSMT in a sample of adults with long-term LVADs.

\section{Methods}

\section{Study Design and Sample}

We analyzed existing data from a multistage instrumentation study ${ }^{16}$ that received Institutional Review Board approval. A total of 189 LVAD patients from various regions of the United States participated in the study, completed in 2015. The inclusion and exclusion criteria, as well as recruitment, consenting and screening process, and data collection procedures employed in the parent study have been published elsewhere. ${ }^{16}$

\section{Data Management and Analyses}

We inspected the data file of the parent study and extracted socio-demographics data, as well as seven variables that are conceptually related to the context, SM process, and outcome dimensions of IFSMT. Variables that comprised the context dimension included self-ratings of cognitive function (general and executive), anxiety, and depression. A single variable, self-efficacy, comprised the SM process dimension, whereas adherence and QOL comprised the proximal and distal outcomes dimension, respectively. The operational definition, measurement, validity, and reliability of measures used for the study variables are summarized in Table $1 .{ }^{16-20}$

Next, we examined the pattern of missing data from the extracted data set. Of the 189 participants, 100 LVAD patients had completed the socio-demographics and seven study variables data needed for analyses (ie, theoretical testing). Subsequently, the final data set $(\mathrm{N}=100)$ was analyzed with descriptive and inferential statistical procedures. Partial least squares path modeling (PLS-PM) ${ }^{21}$ was used to infer the relationships among the IFSMT context, process, and outcome dimensions. Furthermore, we used the bootstrap resampling method to estimate the standard deviations of PLS-PM estimates and test the 
statistical significance of the estimated effect size of the relationship between theoretical dimensions, against a significance criterion of .05 . As previously reported, ${ }^{16}$ we found no significant relationships among socio-demographics (eg, age and gender), clinical (eg, LVAD type and indications) and study variables (eg, cognition and anxiety). Thus, sociodemographics and clinical variables were not included in the PLS-PM analyses. Data were analyzed using IBM SPSS 22.0 and R 3.4.1 Software. ${ }^{22,23}$

\section{Results}

\section{Characteristics of the Sample}

The socio-demographic characteristics of the 100 patients in the present study are summarized in Table 2. Patient age ranged from 20 to 82 years (mean, $52 \pm 13.4$ years). Most patients were White (78\%), male (69\%), educated beyond high school $(72 \%)$, and from the Midwestern (28\%) region of the US. Additionally, $69 \%$ of patients were married, on disability (53\%), and living with a designated caregiver (88\%). Before receiving their LVAD, patients lived with heart failure for an average of $8.1 \pm 6.6$ years. All patients had continuous flow LVAD designs, $86 \%$ with axial flow and $14 \%$ with centrifugal flow. The LVAD indications were bridge-to-transplant $(70 \%)$, destination therapy $(22 \%)$, and bridge-tomyocardial recovery (8\%) with implant duration ranging from 2 to 74 months (mean $20.1 \pm$ 15.6 months).

\section{LVAD SM Variables}

Table 3 shows a descriptive statistics summary characterizing the seven study variables. As shown in this table, the LVAD patients' mean scores of general cognitive function and executive function were similar and slightly lower than normative samples. ${ }^{17}$ Their anxiety and depression respective mean scores were also similar, but were slightly 
higher than normative samples. ${ }^{18,19}$ LVAD care self-efficacy, LVAD care adherence, and QOL mean scores were within normal ranges (Table 3).

Theoretical fit of IFSMT in LVAD SM

The path diagram shown in Figure 2 is a depiction of the quantified relationships among the LVAD SM variables fitted in each dimension of the IFSMT. Results of the PLSPM showed the following forward path relationships among IFSMT dimensions: (a) context was associated with SM process; (b) SM process was associated with proximal outcome; (c) proximal outcome was associated with distal outcome; (d) context was associated with proximal outcome; and (e) context was associated with distal outcome. All of these relationships were significant with effect sizes ranging from small $(\beta=.02)$ to large $(\beta=.60)$.

All relationships shown in the path diagram (Figure 2) are supported by both $p-$ values less than .05 in PLS-PM and factor loadings of latent variables associated with IFSMT dimensions. The factor loadings of the latent variables associated with the context dimension were the following: $50 \%$ (general cognition); $52.5 \%$ (executive function); $84.1 \%$ (anxiety); and $84.2 \%$ (depression). LVAD care self-efficacy, a latent variable associated with the SM process dimension, showed a factor loading of $99.9 \%$. Respective factor loadings for LVAD care adherence and QOL, and latent variables associated with proximal and distal outcome dimensions, were $99.8 \%$ and $100 \%$.

\section{Discussion}

The results of the present study infer the theoretical fit of the IFSMT in LVAD SM, albeit with a small effect $(\beta=.02)$, of the path relationship between proximal (adherence) and distal (QOL) outcome dimensions. We attributed the small effect to the relatively small sample size. However, previous research showed a moderately strong correlation between LVAD care adherence and QOL ( $r=.50)$, and LVAD care adherence was a predictor of

QOL. ${ }^{3,24}$ Research in adults with chronic diseases also demonstrated the significant 
relationship between adherence (ie, medication) and QOL ${ }^{25}$ The moderate effects of the relationships we found between context and process $(\beta=.27)$ and context and proximal outcome $(\beta=.15)$ dimensions concur with much extant findings from other chronic disease populations..$^{8-12,25}$ While there is evidence showing the relationships among cognition, anxiety, depression (context), self-efficacy (process), and adherence (proximal outcome) in chronic diseases and LVADs, ${ }^{3,24-28}$ the relationships among anxiety, depression, and selfefficacy found in the present study are novel findings.

The large effects of the path relationships between context and distal outcome $(\beta=-$ .54) dimensions and SM process and proximal outcome $(\beta=.60)$ dimensions are findings with notable significance. Perhaps more significant is the inverse relationship between context (eg, anxiety) and distal outcome (QOL) dimensions. This finding can be explained by the fact that anxiety and depression are highly prevalent in the LVAD population. ${ }^{29,30}$ As shown in Table 3, our study patients' anxiety and depression scores were slightly worse than the average score of US adults living with the same condition (anxiety and depression). ${ }^{18}$ Remarkably, the coexistence of anxiety and depression, and associated negative influences on QOL, are commonly reported in heart failure studies and from data derived from the LVAD population. ${ }^{29-31}$

The factor loadings for cognitive function (general and executive) further explain the inverse relationship between context and distal outcome dimensions. Cognitive dysfunction is common in heart failure, and its negative effect on the patient's QOL is widely known. Thus, its presence is routinely assessed for pre LVAD implant. ${ }^{3}$ However, there is a paucity of data-based publications involving the cognitive function of adults who are supported by continuous flow LVADs. Two research teams reported the high prevalence of cognitive dysfunction (impairment) in patients implanted with continuous flow LVADs. They found that cognitive impairment is common up to 1-year post LVAD implant, and that such impairment 
is profound in older adults, aged 70 years and older. ${ }^{32,33}$ Comparatively, our sample is relatively younger ( $10 \%$ comprised of patients aged 70 to 82 years). Although we used a different measure than other studies, ${ }^{32,33}$ our measures of general cognition and executive function (Table 3) of our study patients were still slightly worse than the average score of adults in the US with cognitive dysfunction. ${ }^{19}$

Given the known adverse effects of anxiety, depression, and cognitive dysfunction on learning, as well as performing SM tasks accurately and regularly, ${ }^{3,34}$ further research is needed to fully examine these contextual variables. Research is also needed to examine the degree to which the family (eg, caregiver) and healthcare providers' (eg, VAD coordinators) support affect the individual SM outcomes. This triadic research design is crucial to identify the mechanisms or pathways by which the individual SM risk factors can be reduced and managed. According to the IFSMT, ${ }^{8}$ a supportive family or social structure is an essential "protective factor" of effective SM outcomes. Protective SM factors in LVAD can be achieved by the collaborative efforts of patients, family caregivers, and healthcare providers. Early assessment and intervention of the individual (eg, anxiety, cognitive dysfunction) and family (eg, caregiver competence and confidence) SM risk factors are examples of "protective" strategies that can be embedded in mechanical circulatory support programs to optimize LVAD SM outcomes. These strategies can be implemented by VAD nurses/coordinators, who are responsible for providing long-term care and psychoeducational support for LVAD patients and caregivers. ${ }^{6}$

It is worth noting that the relationship between process (self-efficacy) and proximal outcome (adherence) was the largest $(\beta=.60)$ among the path coefficients we found in our study. This finding is expected due to the relatively high self-efficacy and adherence scores (Table 3), which are above the middle point of possible sum scores. ${ }^{16}$ We can infer from our data that a higher level of LVAD care adherence is directly influenced by higher LVAD care 
self-efficacy, parallel to the IFSMT's assumptions. According to the IFSMT, sufficient knowledge, beliefs, and confidence of disease management (self-efficacy) directly affect the results of SM behaviors, such as increased engagement (adherence) in following prescribed treatment regimens. ${ }^{8,15}$ Designing research to elucidate the causality between self-efficacy and adherence in adults with LVADs (bridge-to-heart transplant, destination therapy, or bridge-to-myocardial recovery) would be a logical next step to use the IFSMT framework to advance LVAD SM science (Table 4).

\section{Limitations}

The main limitations of our study included secondary data analyses, the concurrent observational research design, and the convenient sampling method employed in the parent study. The use of self-administered questionnaires, which are a potential source for response bias, ${ }^{16}$ further limits the interpretation of the study findings. This is a particular issue for self-ratings of cognition as depression and anxiety have been shown to influence self-ratings of cognitive impairment. ${ }^{35}$ Furthermore, self-ratings of cognition may not correlate with objective ratings of cognition. ${ }^{36,37}$ Moreover, $88 \%$ of the patients lived with their family caregivers (Table 2). Thus, the possibility of caregivers helping patients complete the questionnaires cannot be ruled out. Finally, the theoretical fit of the data was limited to select conceptual dimensions. Therefore, these limitations prevented us from making definitive conclusions and comprehensive empirical support for the IFSMT in LVAD SM.

\section{Future Directions}

Despite the limitations, we hope that our efforts to initiate empirical support on the IFSMT will stimulate scholarly dialogue in the mechanical circulatory support community, and heighten awareness regarding the current state of the science underpinning the customary practice of LVAD SM. To encourage other investigators and clinicians to move 
the LVAD SM science forward, Table 4 offers several examples of topics that are amendable for research and quality/performance improvement studies aimed at advancing LVAD SM science and practice. These topics are clustered around the IFSMT dimensions, which have been raised in the literature as pertinent variables or factors that may impact LVAD SM outcomes. ${ }^{3-6,14,16,24}$ Future studies should address our study's limitations, as well as explore and expand upon our recommendations in Table 4. Since LVAD SM is actualized by patients in collaboration with family caregivers and VAD nurses/coordinators, ${ }^{3-}$ $6,14,16$ triadic longitudinal research designs will be imperative to explicate the caregiver and coordinators' contributions in LVAD SM outcomes. Equally important are large studies reflective of the LVAD population characteristics (eg, race and education) in the United States covering concepts beyond the present study to establish a solid empirical base and generalizability of the application of IFSMT in LVAD SM.

\section{Conclusion}

Self-rated anxiety, depression, and cognitive dysfunction are individual contextual influences of the LVAD SM process (self-efficacy) and outcomes (adherence and QOL). Our data provide initial evidence corroborating the multidimensionality of SM defined by IFSMT. The IFSMT is a comprehensive framework that can be used for conceptualizing research and clinical scholarly work aimed at advancing the science underpinning LVAD SM practice. Large mechanistic studies are still needed to move the current LVAD SM science from its formative stage to a well-circumscribed knowledge development. The latter is foundational to create and test interventions that will form evidence-based SM practice guidelines designed for preventing complications, reducing healthcare utilizations, and optimizing health and QOL outcomes among adults living with long-term LVADs. 


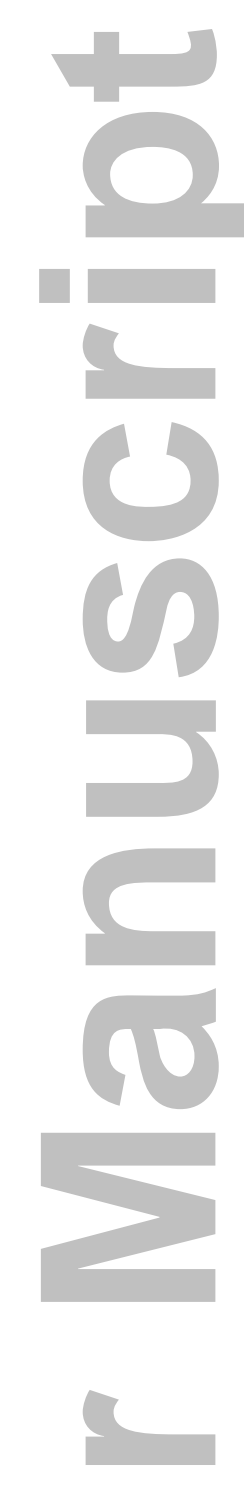

\section{References}

1. Gustafsson F, Rogers JG. Left ventricular assist device therapy in advanced heart failure: patient selection and outcomes. Eur J Heart Fail 2017;19:595-602.

2. Silva Enciso J. Mechanical circulatory support: current status and future directionsProg Cardiovasc Dis 2016;58:444-54.

3. Casida JM, Wu HS, Abshire M, Ghosh B, Yang JJ. Cognition and adherence are selfmanagement factors predicting the quality of life of adults living with a left ventricular assist device. J Heart Lung Transplant 2017;36:325-330.

4. Kato $\mathrm{N}$, Jaarsma $\mathrm{T}$, Ben Gal T. Learning self-care after left ventricular assist device implantation. Curr Heart Fail Rep 2014;11(3):290-8. 
5. Casida J, Wu HS, Harden J, Carie A, Chern J. Evaluation of the psychometric properties of self-efficacy and adherence scales for caregivers of patients with a left ventricular assist device. Prog Transplant 2015;25:116-23.

6. Casida JM, Ilacua J. A survey of nurses in the mechanical circulatory support programs in the United States. Heart Lung. 2011;40:e103-11.

7. Lorig KR. Self-management education: history, definition, outcomes, and mechanisms. Ann Behav Med 2003; 26:1-7.

8. Ryan P, Sawin KJ. The individual and family self-management theory: Background and perspectives on context, process, and outcomes. Nurs Outlook 2009;57:217-225.

9. Grey M, Schulman-Green D, Knafl K, Reynolds NR. A revised self-and family management framework. Nurs Outlook 2015;63:162-70.

10. Grady PA, Gough LL. Self-management: A comprehensive approach to management of chronic conditions. Am J Public Health. 2014; 104: e25-e31.

11. Schulman-Green D, Jaser S, Martin F, et al. Processes of self-management in chronic illness. J Nurs Scholarsh 2012;44:136-44.

12. National Institute of Health. Self-management: Improving the quality of life for individuals with chronic illness. National Institute of Nursing Research website.

https://www.ninr.nih.gov/aboutninr/ninr-mission-and-strategic-plan/themes-selfmanagement. Accessed July 28, 2017.

13. Grey M, Knafl K, Ryan P, Sawin KJ. Differing perspectives on conceptual approaches to self-management. Nurs Outlook 2010; 58: 111-12.

14. Casida JM, Peters RM, Magnan MA. Self-care demands of persons living with an implantable left-ventricular assist device. Res Theory Nurs Pract. 2009;23:279-93. 
5. University of Wisconsin-Milwaukee. Self-management science center - theory. College of Nursing website. https://uwm.edu/nursing/about/centers-institutes/selfmanagement/theory/. Accessed July 28, 2017.

6. Casida J, Wu HS, Harden J, Chern J, Carie A. Development and initial evaluation of the psychometric properties of self-efficacy and adherence scales for patients with a left ventricular assist device. Prog Transplant 2015;25:107-15.

7. National Institute of Neurological Disorders and Stroke (NINDS). User Manual for the Quality of Life in Neurological Disorders (Neuro-QOL) Measures, version 1.0, September 2010.

8. NIH-PROMIS. Anxiety: A Brief Guide to the Anxiety PROMIS Instruments. https://www.assessmentcenter.net/documents/PROMIS\%20Anxiety\%20Scoring\%20Ma nual.pdf. September 2015. Accessed July 28, 2017.

9. NIH-PROMIS. Depression: A Brief Guide to the Depression PROMIS Instruments. https://www.assessmentcenter.net/documents/PROMIS\%20Depression\%20Scoring\%20 Manual.pdf. September 2015. Accessed July 28, 2017.

0. World Health Organization Quality of Life-BREF (WHOQOL-BREF) U. S. Version. http://depts.washington.edu/seaqol/docs/WHOQOL-BREF. January 2014. Accessed July 28, 2017.

1. Tenenhaus M, Espositio VV, Chatelin YM, Lauro C. PLS path modeling. Computational statistics and data analysis 2005;48:159-205.

2. IBM Corporation. IBM SPSS Statistics for Windows Version 22. Armok, NY: IBM Corp; 2013.

3. R Core Team. R: A language and environment for statistical computing. Vienna, Austria: R Foundation for Statistical Computing.https://www.R-project.org/. Accessed August 30, 2017. 
4. Casida JM, Wu HS, Senkiv V, Yang JJ. Self-efficacy and adherence are predictors of quality of life in patients with left ventricular assist devices. J Heart Lung Trans 2016;35:S90-91.

5. Ruppar TM, Cooper PS, Mehr DR, Delgado JM, Dunbar-Jacob JM. Medication adherence interventions improve heart failure mortality and readmission rates: Systematic review and meta-analysis of controlled trials. J Am Heart Assoc 2016;17;5(6). pii: e002606. doi: 10.1161/JAHA.115.002606.

6. Allahverdipour A, AsghariJafarabadi M, Heshmati R, Hashemiparast M. Functional status, anxiety, cardiac self-efficacy, and health beliefs of patients with coronary heart disease. Health Promot Perspect 2013; 3: 217-29.

7. Vellone E, Pancani L, Greco A, Steca P, Riegel B. Self-care confidence may be more important than cognition to influence self-care behaviors in adults with heart failure:

Testing a mediation model. Int J Nurs Stud 2016;60:191-9.

8. Lin MY, Liu MF, Hsu LF, Tsai PS. Effects of self-management on chronic kidney disease: A meta-analysis. Int J Nurs Stud 2017;74:128-137.

9. Reynard AK, Butler RS, McKee MG, Starling RC, Gorodeski EZ. Frequency of depression and anxiety before and after insertion of a continuous flow left ventricular assist device. AM J Cardiol 2014;114:433-40.

0. Bidwell JT, Lyons KS, Mudd JO et al. Quality of life, depression, and anxiety in ventricular assist device therapy. J Cardiovasc Nurs 2016; Nov 2. [Epub ahead of print].

1. Easton K, Coventry P, Lovell K, Carter LVA, Deaton C. Prevalence and measurement of anxiety in samples of patients with heart failure: meta-analysis. J Cardiovasc Nurs 2016; 31:367-79.

2. Bhat G, Yost G, Mahoney E. Cognitive function and left ventricular assist device implantation. J Heart Lung Transplant 2015;34:1398-405. 
3. Fendler TJ, Spertus JA, Gosch KL et al. Incidence and predictors of cognitive decline in patients with left ventricular assist devices. Circ Cardiovasc Qual Outcomes 2015;8:28591.

4. Fredericks S, Lapum J, Lo J. Anxiety, depression, and self-management: a systematic review. Clin Nurs Res 2012;21:411-30.

5. Saffer BY, Lanting SC, Koehle MS, Klonsky ED, Iverson GL. Assessing cognitive impairment using PROMIS ${ }^{\circledR}$ applied cognition-abilities scales in a medical outpatient sample. Psychiatry Res 2015;226:169-72.

6. Meltzer EP, Kapoor A, Fogel J et al. Association of psychological, cognitive, and functional variables with self-reported executive functioning in a sample of nondemented community-dwelling older adults. Appl Neuropsychol Adult 2017;24:364-75.

7. Mohn C, Rund BR. Neurocognitive profile in major depressive disorders: Relationship to symptom level and subjective memory complaints. BMC Psychiatry 2016;16:108.doi: 10.1186/s12888-016-0815-8.

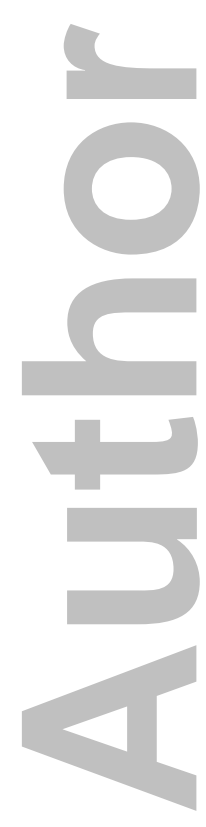


Figure 1: Conceptual elements of the individual and family self-management theory ${ }^{15}$

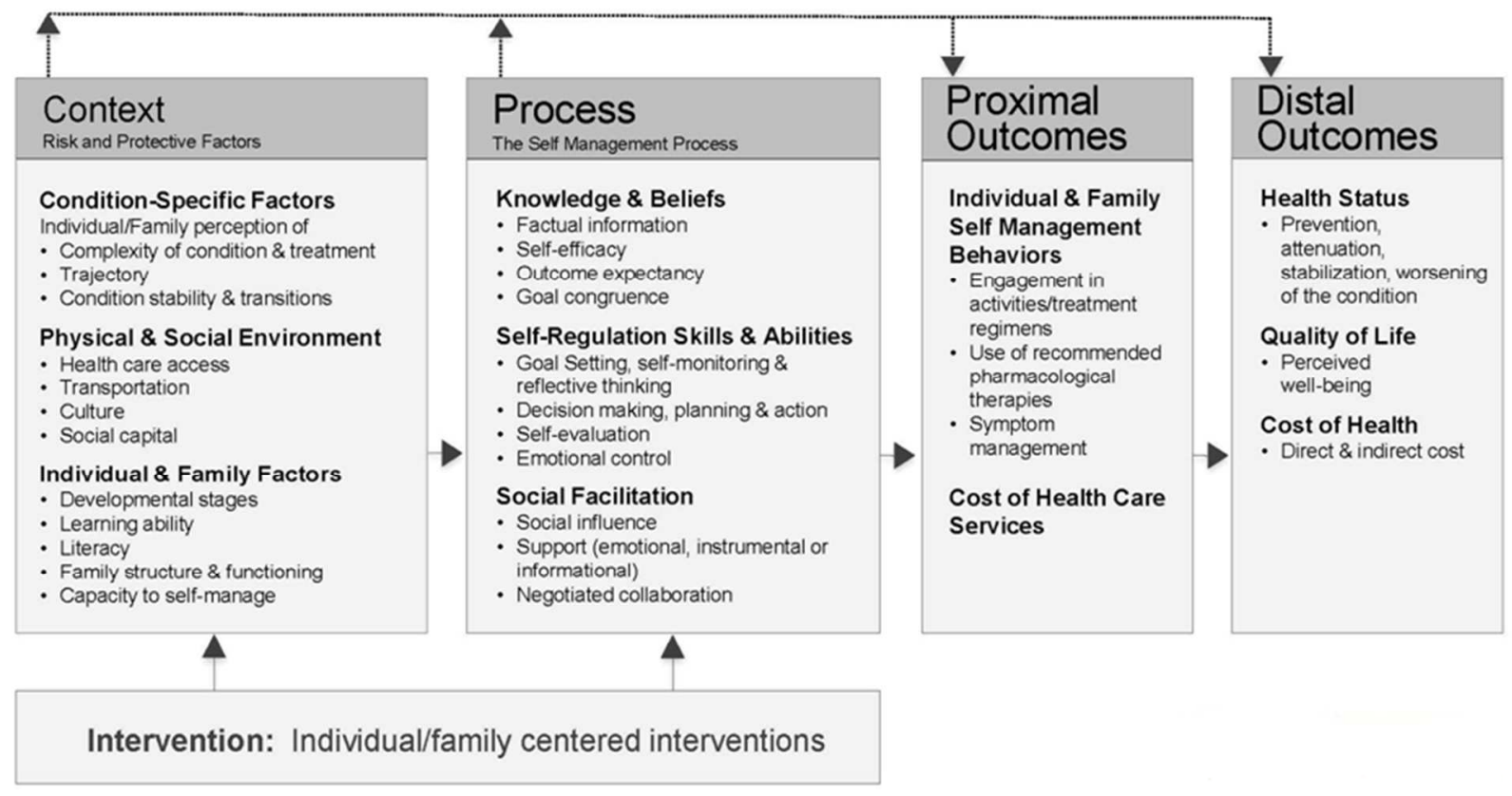

Note: Adopted with authors' permission and the University of Wisconsin-Milwaukee

College of Nursing 
Figure 2: $\quad$ Associations among the context, process, and outcome dimensions of the Individual and Family Self-Management Theory

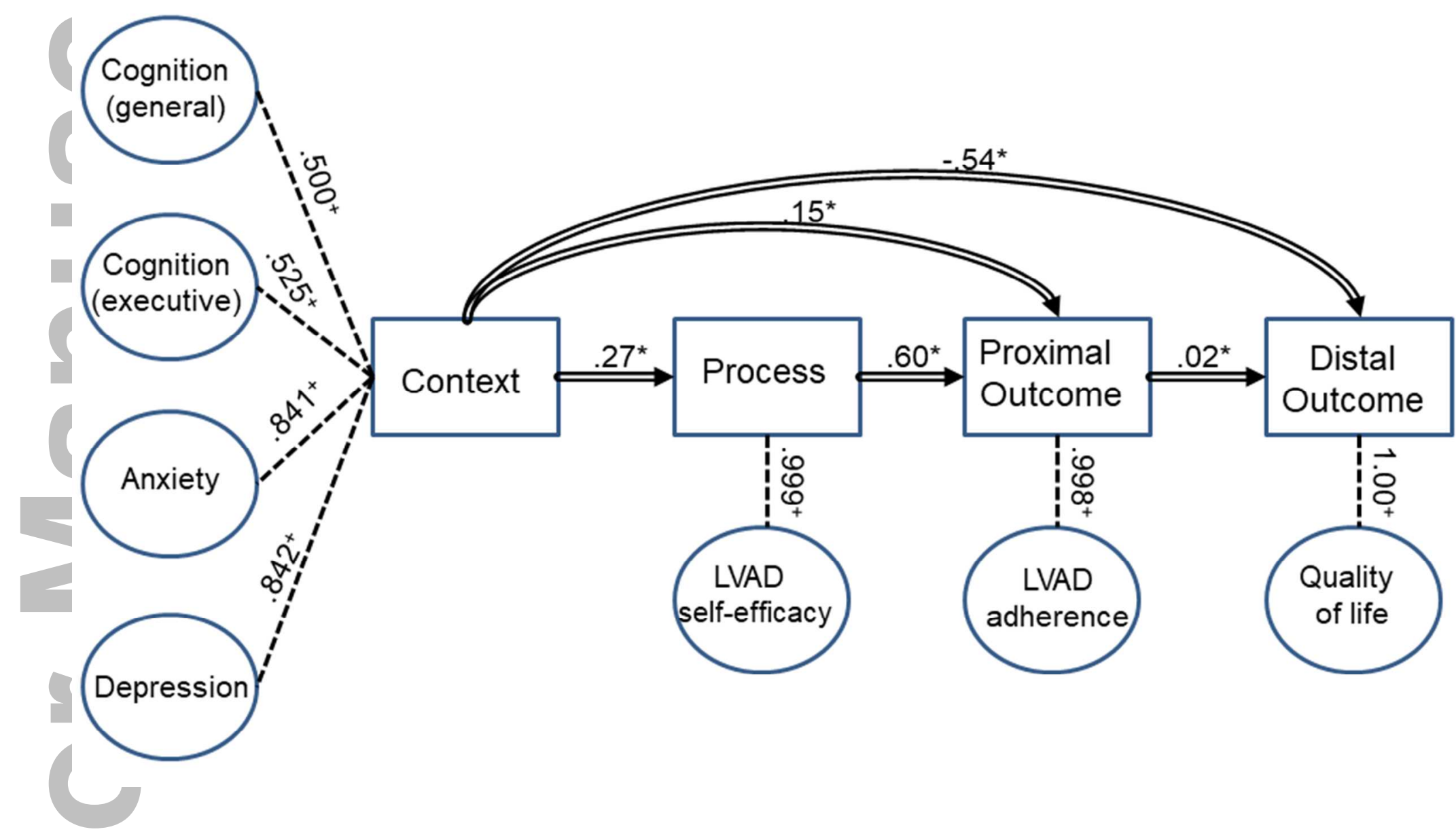

Note: *path coefficients, $p$ values $<.05{ }^{*}$ factor loadings 
Figure 2: $\quad$ Path diagram illustrating the aAssociations among the context, process, and outcome dimensions of the Individual and

Family Self-Management Theory_(IFSMT)

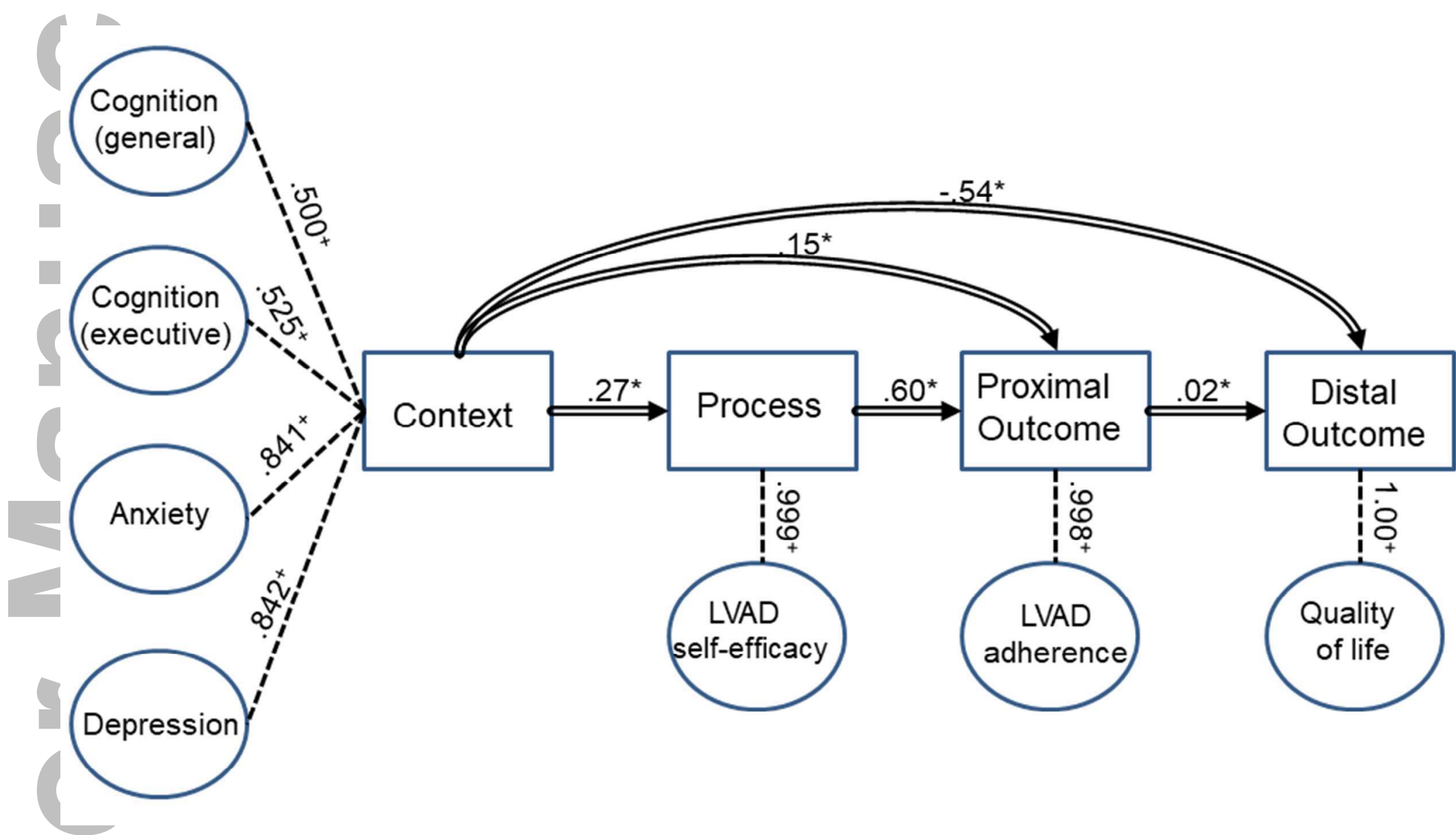

Note: Circles with broken arrows are latent variables (concepts) for each dimension of the IFSMT; solid and broken arrows indicate forward associations between variables; ${ }^{*}$ path coefficients significant at the, $p$ values < .05 level (two-tailed); ${ }^{+}$factor loadings 
Figure 2: $\quad$ Path diagram illustrating the associations among the context, process, and outcome dimensions of the Individual and Family Self-Management Theory (IFSMT)

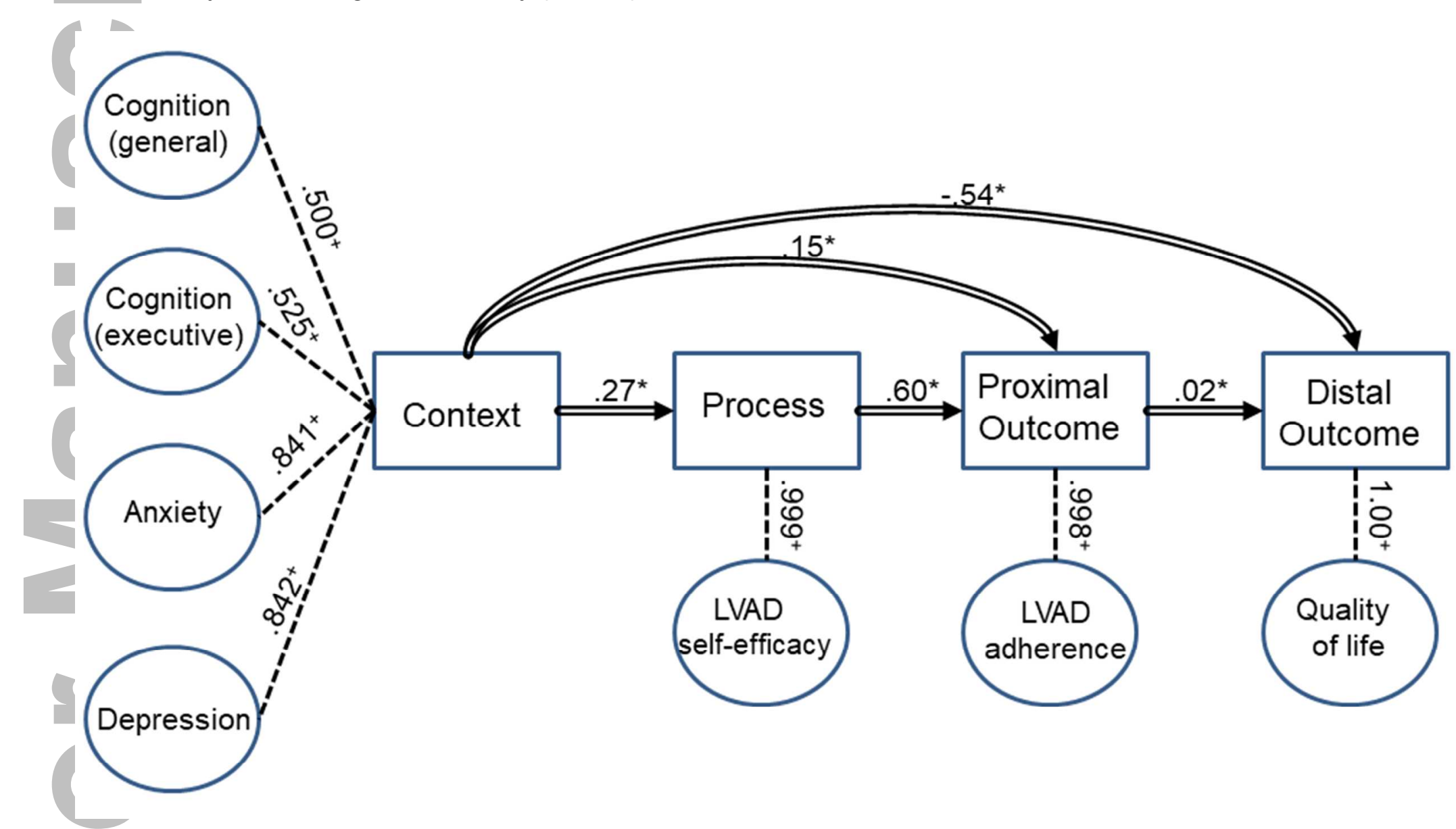

Note: Circles with broken arrows are latent variables (concepts) for each dimension of the IFSMT; solid and broken arrows indicate forward associations between variables; *path coefficients significant at the .05 level (two-tailed); ${ }^{\text {factor loadings }}$ 
Table 1: Study variables measurements and psychometric properties ${ }^{16-20}$

IFSMT Concept/Variable Measures, Items and Response

Dimensions
Uses and Scores
Reliability and Validity
General cognitive function

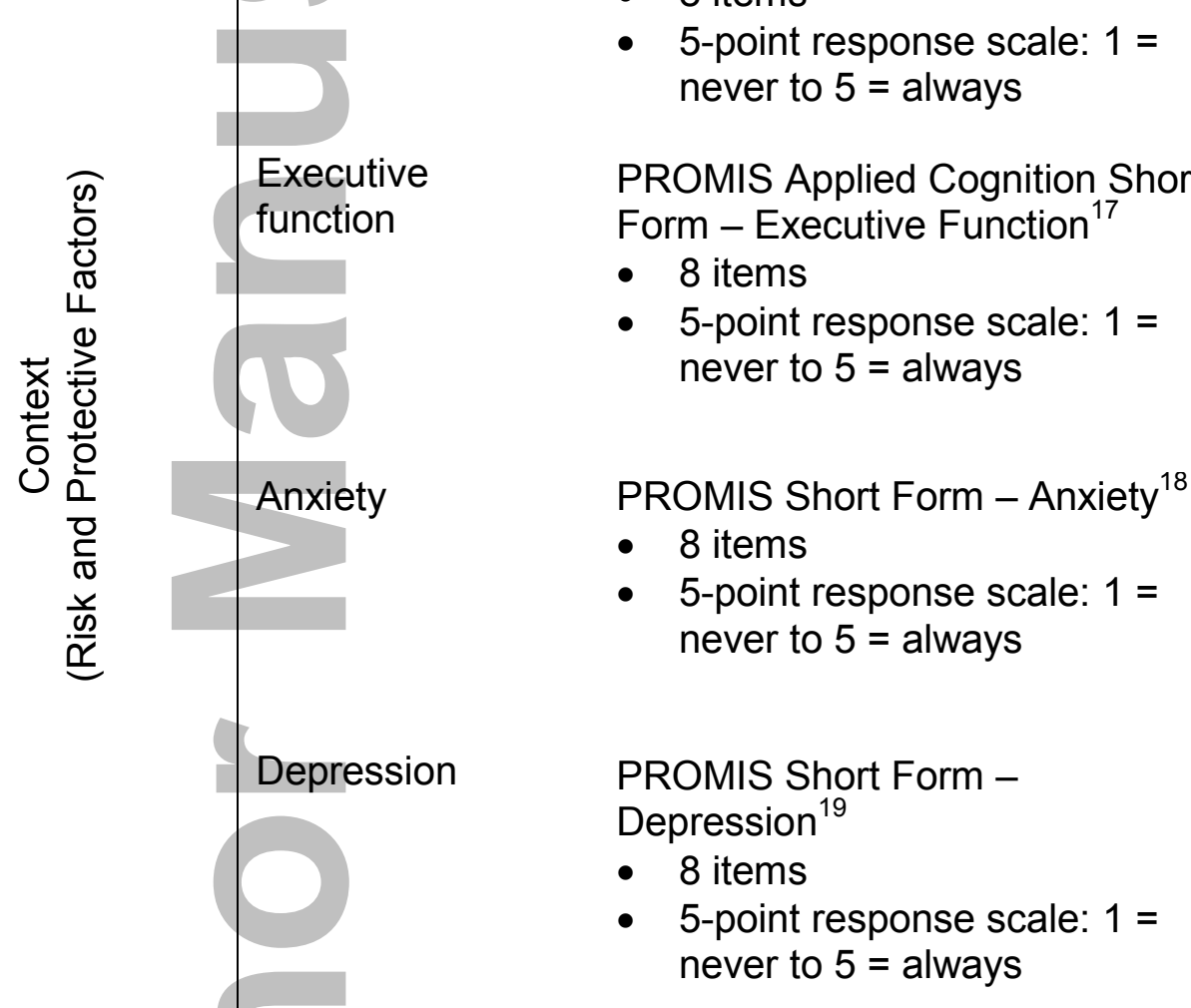

Assesses patient's perceptions of his/her general cognitive concerns such as memory and attention.

Higher mean t-score signifies the concept (ie, general cognitive function) being measured.

Assesses patient's perceptions of his/her difficulties in applying cognitive abilities to daily tasks such as planning and learning.

Higher mean t-score signifies the concept (ie, executive function) being measured.

Assesses the universal symptoms of anxiety such as fear, nervousness, and dizziness.

Higher mean t-score signifies more of the concept (ie, anxiety) being measured.

Assesses the universal symptoms of depression such as feeling worthless and loss of interest.

Higher mean t-score signifies more of the concept (ie, anxiety) being measured.
Cronbach $\alpha=.89$

PROMIS Standards of Psychometric Testing

Chronbach $\alpha=.96$

PROMIS Standards of Psychometric Testing

Cronbach $\alpha=.94$

PROMIS Standards of Psychometric Testing

Chronbach $\alpha=.96$

PROMIS Standards of Psychometric Testing 


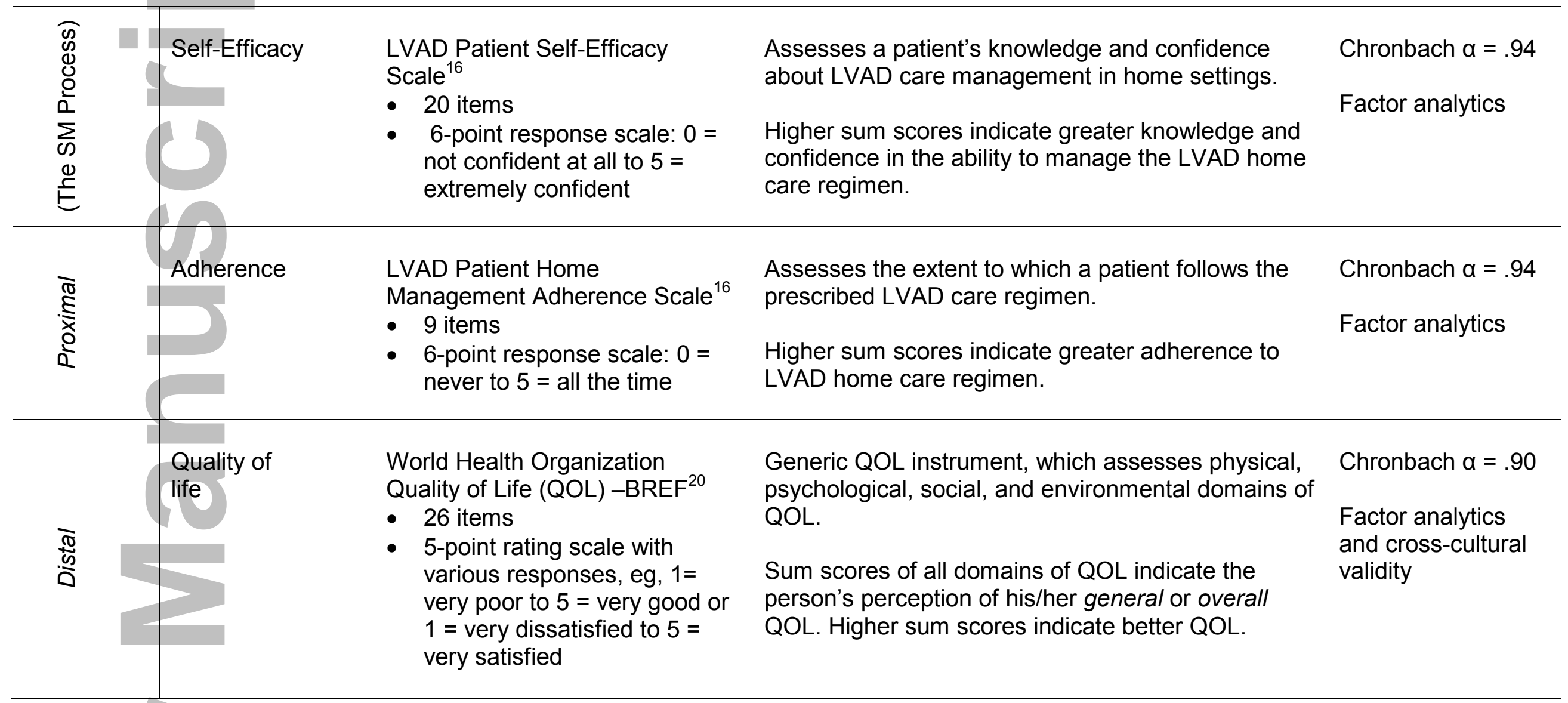

Note: PROMIS - Patient-Reported Outcomes Measurement Information System 
Table 2: Socio-demographic Characteristics of the Sample

\begin{tabular}{|c|c|c|c|}
\hline Characteristic & $\mathrm{n}(\%)^{*}$ & Characteristic & $n(\%)^{*}$ \\
\hline Gender: & & Marital Status: & \\
\hline Male & $69(69)$ & Married & $69(69)$ \\
\hline Female & $31(31)$ & Single & $31(31)$ \\
\hline & & Divorced & $8(8)$ \\
\hline White & $78(78)$ & Employment: & \\
\hline Black & $15(15)$ & Full-time & $15(15)$ \\
\hline Asian & $2(2)$ & Part-time & $5(5)$ \\
\hline Mixed & $3(3)$ & Retired & $21(21)$ \\
\hline Native American & $1(1)$ & Disability & $53(53)$ \\
\hline Hispanic non-white & $1(1)$ & Unemployed & $6(6)$ \\
\hline Region: & & Designated Caregivers: & \\
\hline Northeast & $25(25)$ & Yes & $88(88)$ \\
\hline Midwest & $28(28)$ & No & $12(12)$ \\
\hline Southeast & $14(14)$ & & \\
\hline Southwest & $11(11)$ & & \\
\hline West & $9(9)$ & & \\
\hline \multicolumn{4}{|l|}{ Education: } \\
\hline Less than high school & $5(5)$ & & \\
\hline High school & $23(23)$ & & \\
\hline Some college & $43(43)$ & & \\
\hline College and higher & $29(29)$ & & \\
\hline
\end{tabular}

Note: ${ }^{*}$ Due to missing data not all percentages total 100 
Table 3: Summary statistics of study variables

\begin{tabular}{lcc}
\hline \multicolumn{1}{c}{ Measures and Norms } & Ranges & $\begin{array}{c}\text { Mean and Standard } \\
\text { Deviation }\end{array}$ \\
\hline $\begin{array}{l}\text { Cognitive Function - General } \\
\text { Mean }(t): 50 \pm 10\end{array}$ & $31-59.3$ & $47.4 \pm 8.0$ \\
Cognitive Function - Executive & $19.5-57.6$ & $47.9 \pm 8.7$ \\
Mean $(t): 50 \pm 10$ & & \\
Anxiety & & \\
Mean $(t): 50 \pm 10$ & $37.1-70$ & $51.0 \pm 9.0$ \\
Depression & & $86.76 \pm 14.5$ \\
Mean $(t): 50 \pm 10$ & $38.2-72$ & $39.0 \pm 6.8$ \\
LVAD Patient Self-Efficacy & $11-100$ & \\
Sum: 0 to 100 & & \\
LVAD Patient Home Management & $7-45$ & \\
Adherence & & \\
Sum: 0 to 45 & & \\
World Health Organization & & \\
Quality of Life-BREF & & \\
Sum: 0 to 100 & & \\
\hline
\end{tabular}


Recommendations for future research in LVAD SM guided by the Individual and Family Self-Management Theory (IFSMT)

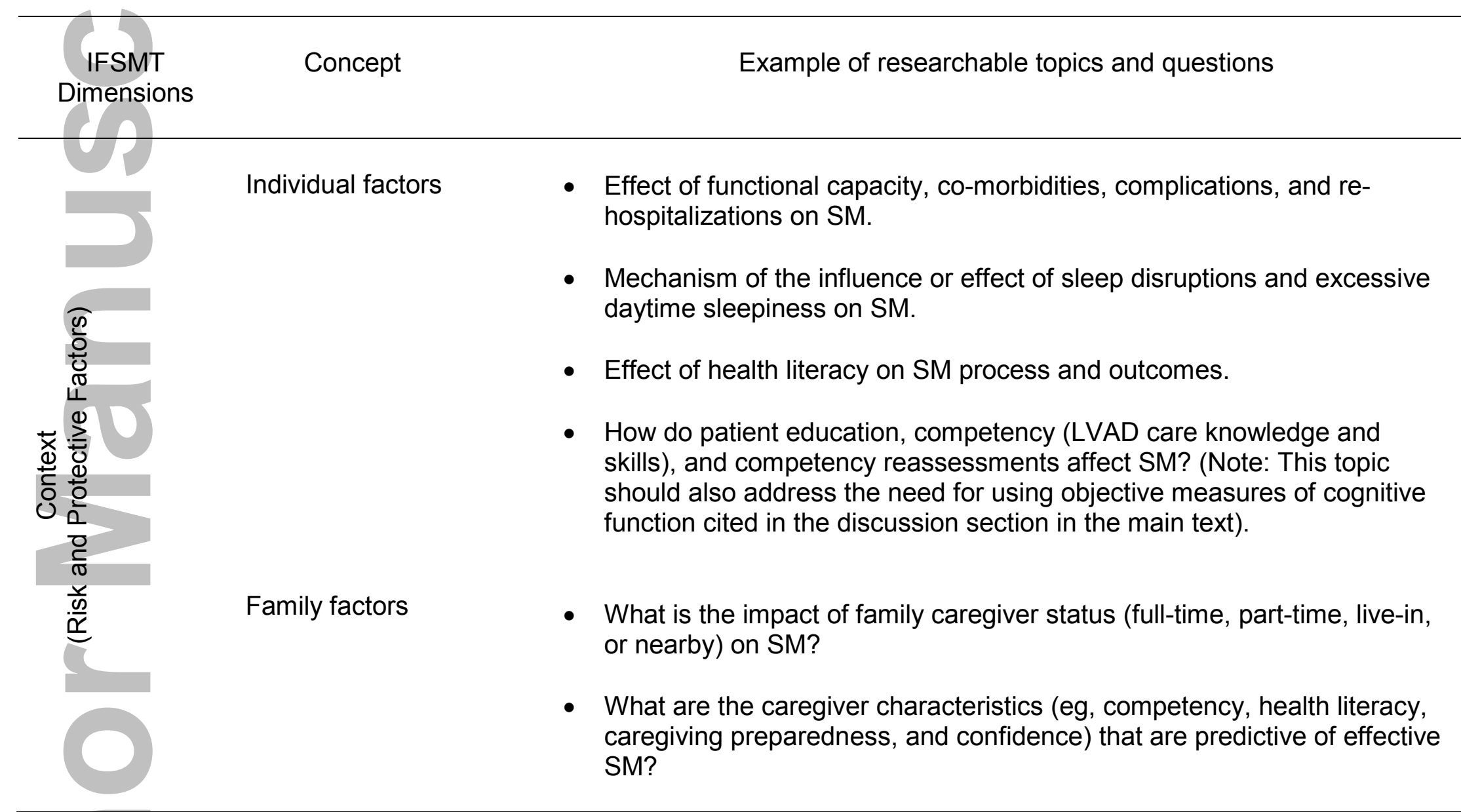


Knowledge and beliefs

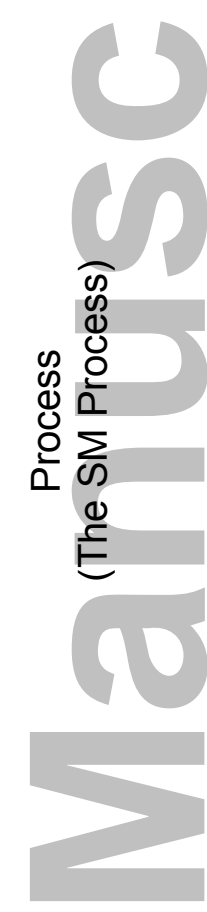

Self-regulation skills and abilities

Social facilitation
- Intervention to optimize LVAD care self-efficacy.

- Explore interventions designed for individual goal setting and outcome expectations (eg, goals for managing LVAD flows specific to the device and individual).

- Develop and test technology (eg, mobile phone app) that are easy and efficient for self-monitoring of LVAD parameters (eg, flow, power) and heart failure symptoms, etc., and features that provide feedback and coach how to manage abnormal parameters and symptoms.

- Intervention to address cognitive difficulties, anxiety, and/or depression and their impact on SM outcomes (can be classified as individual/context SM risk factor).

- Effect of the role of VAD nurses or coordinators (eg, frequent follow up and psychoeducational support) on SM outcomes.

- What VAD care team (eg, skill mix) characteristics are predictive of effective SM outcomes?

- Intervention to improve adherence on LVAD-specific care, medications, diet, and physical activity.

- Apply health promotion, risk reduction, and symptom management interventions tested in heart failure patients with cardiac devices (eg, pacemakers, defibrillators) or post cardiac surgery.

- Hospital re-admissions

- Unscheduled emergency room and clinic visits 
Health status

Quality of life

Cost
- LVAD-specific and related complications, co-morbidity, functional status, frailty and mortality.

- Use of generic and LVAD-specific QOLmeasures

- Cost of technology used for LVAD SM and health care providers' cost supporting LVAD SM 
Recommendations for future research in LVAD SM guided by the Individual and Family Self-Management Theory (IFSMT)

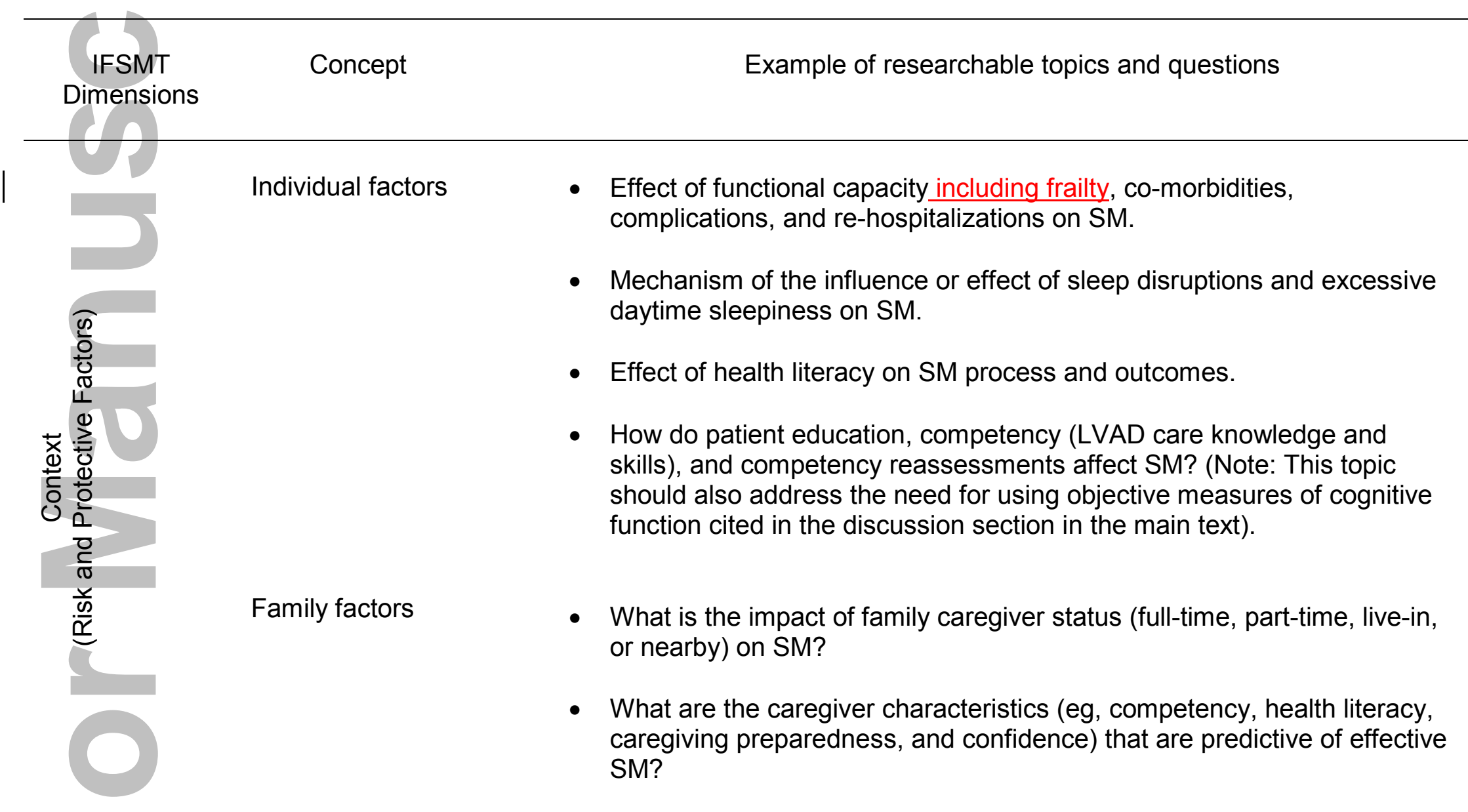


Knowledge and beliefs

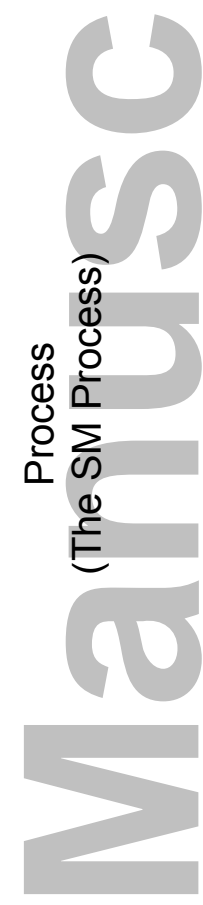

Self-regulation skills and abilities

Social facilitation

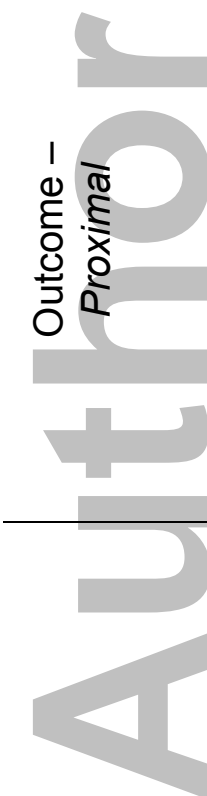

Individual and family selfmanagement behaviors outcomes

Healthcare utilizations
- Intervention to optimize LVAD care self-efficacy.

- Explore interventions designed for individual goal setting and outcome expectations (eg, goals for managing LVAD flows specific to the device and individual).

- Develop and test technology (eg, mobile phone app) that are easy and efficient for self-monitoring of LVAD parameters (eg, flow, power) and heart failure symptoms, etc., and features that provide feedback and coach how to manage abnormal parameters and symptoms.

- Intervention to address cognitive difficulties, anxiety, and/or depression and their impact on SM outcomes (can be classified as individual/context SM risk factor).

- Effect of the role of VAD nurses or coordinators (eg, frequent follow up and psychoeducational support) on SM outcomes.

- What VAD care team (eg, skill mix) characteristics are predictive of effective SM outcomes?

- Intervention to improve adherence on LVAD-specific care, medications, diet, and physical activity.

- Apply health promotion, risk reduction, and symptom management interventions tested in heart failure patients with cardiac devices (eg, pacemakers, defibrillators) or post cardiac surgery.

- Hospital re-admissions

- Unscheduled emergency room and clinic visits 
Health status

Quality of life

Cost
- LVAD-specific and related complications, co-morbidity, functional status, frailty and mortality.

- Use of generic and LVAD-specific QOLmeasures

- Cost of technology used for LVAD SM and health care providers' cost supporting LVAD SM 
Recommendations for future research in LVAD SM guided by the Individual and Family Self-Management Theory (IFSMT)

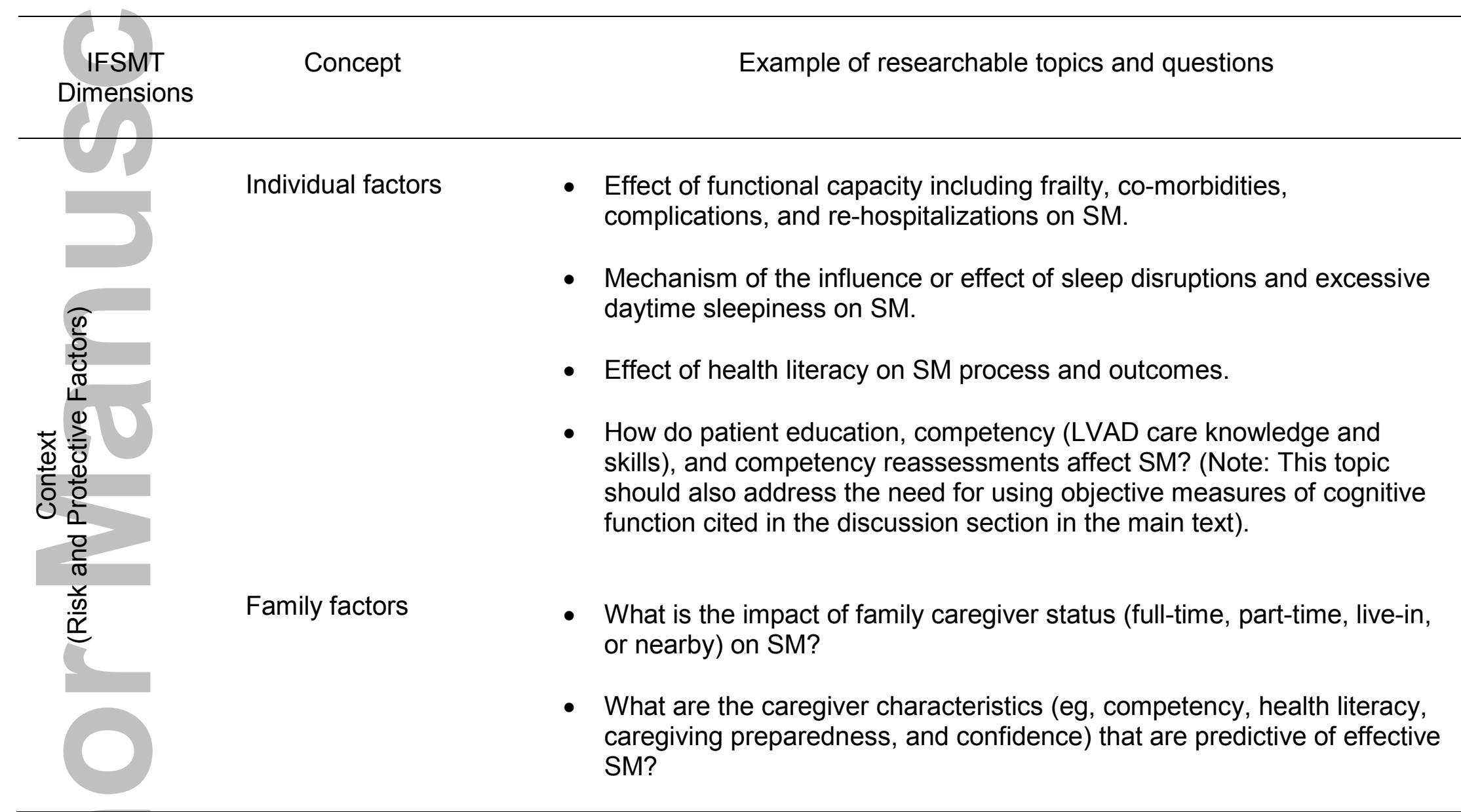


Knowledge and beliefs

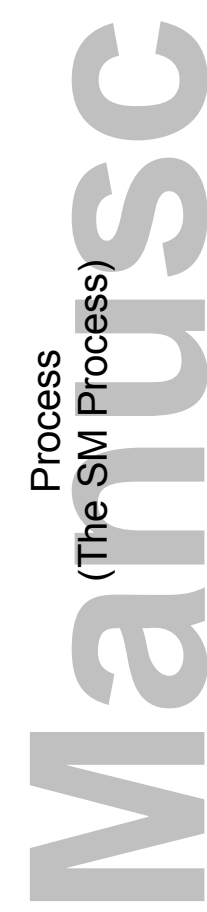

Self-regulation skills and abilities

Social facilitation
- Intervention to optimize LVAD care self-efficacy.

- Explore interventions designed for individual goal setting and outcome expectations (eg, goals for managing LVAD flows specific to the device and individual).

- Develop and test technology (eg, mobile phone app) that are easy and efficient for self-monitoring of LVAD parameters (eg, flow, power) and heart failure symptoms, etc., and features that provide feedback and coach how to manage abnormal parameters and symptoms.

- Intervention to address cognitive difficulties, anxiety, and/or depression and their impact on SM outcomes (can be classified as individual/context SM risk factor).

- Effect of the role of VAD nurses or coordinators (eg, frequent follow up and psychoeducational support) on SM outcomes.

- What VAD care team (eg, skill mix) characteristics are predictive of effective SM outcomes?

- Intervention to improve adherence on LVAD-specific care, medications, diet, and physical activity.

- Apply health promotion, risk reduction, and symptom management interventions tested in heart failure patients with cardiac devices (eg, pacemakers, defibrillators) or post cardiac surgery.

- Hospital re-admissions

- Unscheduled emergency room and clinic visits 
Health status

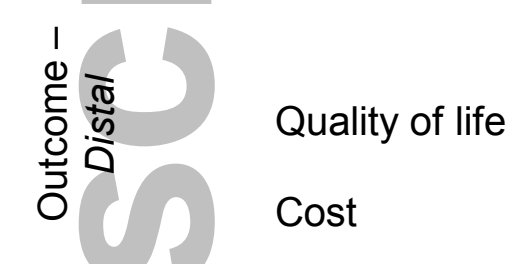

- LVAD-specific and related complications, co-morbidity, functional status, frailty and mortality.

- Use of generic and LVAD-specific QOLmeasures

- Cost of technology used for LVAD SM and health care providers' cost supporting LVAD SM 